\title{
Was bringt die Hypo-Schulung?
}

\author{
Hypoglykämien sind die am meisten gefürchtete \\ Komplikation der Diabetestherapie. Sie verschlech- \\ tern die Prognose, da sie kardiovaskuläre Ereignisse \\ und vitale bedrohliche Herzrhythmusstörungen in- \\ duzieren können, und begünstigen Demenz.
}

Das oberste Ziel einer antidiabetischen Therapie muss die Vermeidung von schweren Hypoglykämien sein. Dies erfordert neben der Medikamentenwahl auch die adäquate Schulung, ein individuelles Training und die Festlegung individueller Therapieziele unter Berücksichtigung der Komorbiditäten.

In einem komplexen Wechselspiel versucht der Organismus, den Blutzucker (BZ) zwischen 3,9 und 5,5 mmol/l (70-99 mg/ dl) konstant zu halten. „Sinkt der Wert darunter, kommt es zunächst zu autonomen und dann zu neuroglykopenischen Symptomen“, so Dr. Christof Kloos von der Medizinischen Uniklinik in Jena. Die autonomen Symptome wie Schwitzen, Tachykardie und Tremor werden durch Sympathikusaktivierung induziert, bevor die Glykopenie zu kognitiven Einschränkungen mit Verwirrtheit, Seh- und Sprach- sowie Bewusstseinsstörungen bis hin zum Koma führt. Auf hormoneller Ebene wird die endogene Insulinproduktion supprimiert, vermehrt Glukagon und Adrenalin freigesetzt und darüber die intrahepatische Glukoneogenese stimuliert und die periphere Glukoseaufnahme gehemmt.

Eine einheitliche Definition der Hypoglykämie gibt es bisher nicht. „Auch ist die Schwelle, ab wann ein zu niedriger Blutzuckerwert potenziell schädlich ist, individuell sehr unterschiedlich", so Kloos. Bei einer vorhergehenden Hypoglykämie sei sie niedriger, bei einer chronischen Hyperglykämie höher. Im Allgemeinen liege der BZ bei einer symptomatischen Hypoglykämie $<3,1 \mathrm{mmol} / \mathrm{l}(<56 \mathrm{mg} / \mathrm{dl})$. ,Wenn Hilfe durch eine andere Person nötig ist, liegt immer eine schwere Hypoglykämie vor", so Kloos.

Sie erfordere die i.v. Gabe von Glukose bzw. die i. m. oder s.c. Applikation von Glukagon. Bei einer leichten Hypoglykämie könne der Patient sich selbst durch Zuckeraufnahme therapie- ren. Schwere Hypoglykämien seien heute ein sehr seltenes Ereignis, wobei mit einer Rate von 0,04 Hypoglykämien pro Jahr Typ-1-Diabetiker häufiger betroffen sind. Als Risikofaktoren gelten symptomatische Hypoglykämien in der Vorgeschichte, chronische Nierenschwäche, Demenz/kognitive Funktionsstörungen, geriatrischer Zustand, Diabetesdauer $>10$ Jahre, BMI < $22 \mathrm{~kg} / \mathrm{m}^{2}$, autonome Neuropathie bzw. Gastroparese, Polypharmazie, Hypoglykämie-Wahrnehmungsstörung und unzureichende Diabetesschulung.

\section{Daten aus der Versorgungsforschung}

Aus Abrechnungsdaten von AOK-Versicherten mit Typ-2-Diabetes wurde die Inzidenz schwerer Hypoglykämien unter Berücksichtigung der antihyperglykämischen Therapie im Versorgungsalltag erfasst. Dabei zeigte sich eine leichte Zunahme der Inzidenz von $0,004 \%$ im Jahr 2006 auf 0,005\% im Jahr 2011, wobei die Personen mit schwerer Hypoglykämie 2011 etwas älter waren und häufiger eine Nephropathie hatten als 2006.

Unter Sulfonylharnstoffen, Humaninsulin und Kombinationsinsulin wurden Hypoglykämien deutlich seltener dokumentiert, Mit Analoginsulinenaber öfter, was aber nur der Änderungen der Verordnungshäufigkeit geschuldet ist. Die Daten zeigen also, dass das Risiko für schwere Hypoglykämien nicht nur durch des Verordnungsverhalten reduziert werden kann. Das $\mathrm{HbA}_{1 \mathrm{c}}$-Ziel und die Intensität der glukosesenkenden Therapie dürfte wichtiger sein, zumal vorrangig Ältere mit Nierenschwäche betroffen sind.

Untersuchungen ergaben, dass das Risiko für Hypoglykämie bei Typ-1-Diabetikern durch strukturierte Schulung deutlich gesenkt werden kann. Durch besondere Schulungsprogramme kann auch die Hypoglykämiewahrnehmung verbessert werden. Für Typ-2-Diabetiker, bei denen schwere Hypoglykämien sehr selten auftreten, konnte aber bisher nicht belegt werden, dass eine Schulung das Hypoglykämierisiko senkt.

Dr. med. Peter Stiefelhagen

Quelle: 51. DDG-Jahrestagung, 06.05.2016 in Berlin

\section{Hier steht eine Anzeige.}

